\title{
Anotaciones sobre bienestar animal en zoológicos
}

\author{
Annotations about animal welfare in zoos
}

DE LA OSSA V, JAIME ${ }^{1 *}$ Ph.D.

${ }^{1}$ Universidad de Sucre, Facultad de Ciencias Agropecuarias, Grupo de Investigación en Biodiversidad Tropical, Colombia.

\section{Key words:}

Animal welfare; human care; wildlife; conservation.

\section{Palabras Clave:}

Bienestar animal; cuidado humano; fauna silvestre; conservación.

\section{Abstract}

The present works performed a general analysis about some important aspects that have relationship with the behavior and the well-being of animals under the human care and confined in zoological and aquariums, in accordance with the principles of animal welfare they should have good conditions of life, healthy, comfortable, safe, able to express innate behavior, and if it is not suffering from unpleasant states such as pain, fear, and distress. And the other hand, aspects related with the bioethics and the paper of the zoos in the ex situ conservation and the environmental education are addressed.

\section{Resumen}

El presente trabajo hace un análisis general sobre algunos aspectos importantes que tienen relación con el comportamiento y el bienestar de animales bajo cuidado humano en zoológicos y acuarios, de acuerdo con los principios en esta materia se entiende que deben contar con buenas condiciones de vida, estado saludable, confortable, seguro y con capacidad para expresar su comportamiento innato, sin sufrimiento o estados desagradables, tales como dolor, miedo y angustia. De otro lado, se analizan aspectos relacionados con la bioética y el papel de los zoológicos en la conservación ex situ y en la educación ambiental son abordados. 


\section{Introducción}

$<<$ Nadie niega que cada época modula su propia ética de manera que hay un pensamiento moral subordinado a los cambios históricos a partir del cual, en un determinado momento, se acepten las ejecuciones públicas, las corridas de toros o las luchas de gladiadores. Pero no podemos anclarnos en una moral diacrónica, sino en una sincrónica donde el elemento de comparación sean los avances sociales del tiempo que nos ha tocado vivir>> (IVANOVIC BARBEITO (2011)

De acuerdo con el Decreto 1608 de 1978 y la Ley 611 de 2000 se denomina Fauna Silvestre y Acuática al conjunto de organismos vivos de especies animales terrestres y acuáticas, que no han sido objeto de domesticación, mejoramiento genético, cría regular o que han regresado a su estado salvaje. Esta definición contempla a los anfibios, reptiles, aves y mamíferos colombianos no domesticados y demás especies que cumplen el ciclo total de vida dentro del agua, que por definición son recursos hidrobiológicos excluyendo en consecuencia a todos los organismos acuáticos que son aprovechados por el hombre y que se les considera como recurso pesqueros(Ley 13 de 1990).

Existen, además otras consideraciones, que para el caso deberán ser tenidas en cuenta:

Fauna amansada, son individuos de especies de fauna silvestre que han sufrido un proceso de humanización, con comportamientos condicionados y reacciones manipuladas por quienes los mantienen cautivos (CORPONARIÑO, 2016). En esta categoría se encuentran: muchos especímenes de psitácidos, quelonios, primates no humanos, aves canoras, entre otros, que con predilección son mantenidas en calidad de animales de compañía; esta fauna está protegida por la legislación colombiana y es decomisable por las autoridades competentes.

Fauna doméstica: son todos los animales pertenecientes a especies que han sido producto de cría, levante regular o mejoramiento genético y que le han servido incondicionalmente al ser humano a través del tiempo; dentro de estos se tienen, por ejemplo: vacas, caballos, perros, gatos, ovejas, gallinas, pavos, entre otros. Es de anotar que las autoridades ambientales no pueden adelantar decomisos sobre estas especies pero existe para su protección la Ley 84 de 1989 <<Estatuto de Protección a los Animales >>.

Fauna exótica: se considera dentro de esta clasificación todas aquellas especies cuyo origen y evolución están por fuera de los límites físicos del territorio patrio y para tal efecto pueden ser silvestres o domésticas. La introducción a Colombia de este tipo de fauna, tales como: tigres de Bengala, leones, jirafas, chimpancés, orangutanes, se rige de acuerdo con la Ley 99 del 93 articulo 5 numeral 21, Resolución 1367 de diciembre 29 de 2000 de Minambiente y Decreto 1608 de 1978.

Manejo de fauna silvestre ex situ, es decir fuera de su hábitat natural, entre las diferentes modalidades de conservación ex situ para fauna silvestre se tienen los centros de tenencia y manejo tales como: zoológicos, centros de rescate, centros de tránsito, zoocriaderos y museos (VÁLDES, 2008).

Manejo de fauna silvestre in situ, hace referencia a la permanencia dentro del hábitat natural; los procesos de conservación in situ propenden por el mantenimiento de las poblaciones naturales dentro de sus hábitats (BECERRA, 2003). Se tiene como centros de conservación in situ: bosques naturales, áreas protegidas en sus diversas categorías.

De acuerdo con la UNESCO (1977), en la Declaración Universal de los Derechos de los Animales se puede indicar de forma pertinente para este escrito, lo siguiente:

Artículo $1^{\circ}$ - Todos los animales nacen iguales ante la vida y tienen los mismos derechos a la existencia.

Artículo $2^{\circ}$, literal a) Todo animal tiene derecho a ser respetado; literal c) Todos los animales tienen derecho a la atención, a los cuidados y a la protección del hombre.

Artículo $3^{\circ}$, literal a) Ningún animal será sometido a malos tratos ni a actos crueles.

Artículo $4^{\circ}$, literal a) Todo animal perteneciente a una especie salvaje, tiene derecho a vivir en 
libertad en su propio ambiente natural, terrestre, aéreo o acuático y a reproducirse.

Artículo $8^{\circ}$, literal a) La experimentación animal que implique sufrimiento físico o psicológico es incompatible con los derechos del animal, ya se trate de experimentos médicos, científicos, comerciales, o de cualquier otra forma de experimentación.

Artículo $10^{\circ}$, literal a) Ningún animal será explotado para esparcimiento del hombre; literal b) Las exhibiciones de animales y los espectáculos que se sirvan de ellos son incompatibles con la dignidad del animal.

Plantea IVANOVIC BARBEITO (2011), que el punto filosófico de partida del animalismo, se basa en una sencilla premisa: si un ser puede sentir miedo, felicidad o tristeza, no existe justificación alguna para negarse a considerar esos sentimientos primarios, solo porque no puedan ser expresados de forma verbal o escrita. Por ejemplo, todos los mamíferos compartimos un cerebro límbico que interpreta de forma similar dolor y placer (IVANOVIC BARBEITO, 2013). Si los animales humanos nos consideramos agentes morales y tenemos en cuenta que los animales no humanos no pueden defenderse por sí mismos (IVANOVIC BARBEITO, 2011).

No existe justificación alguna para negarse a considerar que sentimientos como el miedo, la felicidad o la tristeza, son también propios de otras especies, aunque no puedan expresarlos de forma escrita o hablada. Los animales carecen de un lenguaje elaborado para verbalizar su sufrimiento; pero, sin embargo, todos los mamíferos compartimos un cerebro límbico que interpreta de forma similar dolor y placer. Si los animales humanos nos consideramos agentes morales y tenemos en cuenta que los animales no humanos no pueden defenderse por sí mismos, es nuestro deber el asumir la responsabilidad de protegerlos (IVANOVIC BARBEITO, 2011).

A manera de ejemplo y con el fin de tener un acercamiento a la situación ética de los animales, en cuanto hace referencia a su protección, se puede vincular lo afirmado por IVANOVIC BARBEITO (2011) con la crisis ambiental que se vive y que quiérase o no se relaciona; plantea, que aunque se deje de lado toda consideración ética sobre el trato que se da a los animales y se les tenga como si fuesen máquinas de producción, se tendría un escenario adecuado para discutir su papel en los problemas medioambientales que nos afectan. De acuerdo con los datos globales, las emisiones de gases de efecto invernadero derivados de la cría de ganado, superan porcentualmente las emisiones de la industria del transporte, ambas sobrepasan el $30 \%$ del total (TUBIELLO et al., 2014). Igualmente se tiene, que el número de animales producidos para consumo humano también representa un peligro para la biodiversidad de la Tierra; el ganado constituye un $20 \%$ del total de la biomasa animal terrestre y la superficie que ocupa actualmente fue antes hábitat de especies silvestres (WINT y ROBINSON, 2007; TUBIELLO et al., 2013). La situación desde una perspectiva bioética muestra el desequilibrio al que se ha llegado con la deshumanización del trato hacia los animales y la insostenibilidad del manejo de los recursos naturales del planeta, situación crítica que negativamente actúa en sinergia con el cambio climático.

A manera de conclusión y con acogimiento a lo expresado por IVANOVIC BARBEITO (2011), hay que señalar que los animales no son objetos, aunque sean tratados como tales en el modelo antropocéntrico y productivista imperante. El antropocentrismo considera moralmente relevante sólo al ser humano, mientras que la Naturaleza y los animales sólo tienen valor en tanto que sean portadores de utilidad en el sentido económico.

\section{Un acercamiento epistemológico}

Como parte fundamental del enfoque que debe dársele a los parques zoológicos y acuarios, debe ser entendida la realidad filosófica que los rodea y que de forma acertada VASTA (2013) señala al decir, que: durante el Siglo XVII, Descartes cristalizó el pensamiento que dividía el cuerpo del alma y el hombre de la naturaleza, y se formalizó la idea de objetividad: el sujeto debía abstraerse de su corporalidad para conocer y debía penetrar la naturaleza para comprenderla. (Res cognitas vs. Res extensa). Esta acción abrió la brecha que alejó cada vez más fuertemente a hombres de animales; al hacer una división absoluta entre el alma y el cuerpo, el filósofo francés legó el cuerpo 
a las leyes de la física y la mecánica, y, como los animales no tenían alma, quedaron reducidos al modelo mecánico.

Continúa esta misma autora, expresando: el surgimiento del museo y el jardín zoológico se produjo dentro de este nuevo contexto. En términos generales, tanto el espacio del museo como el espacio del zoológico se estructuraron sobre una ideología de la visibilidad -la visión como paradigma central de la adquisición del saber en occidente-, con una propuesta de sentido articulada bajo la trilogía Recorrido-OrientacionOrden; y asimismo se constituyeron en una operación ideológico-social oculta tras las ideas de la difusión de la ciencia -heredadas de la filosofía de las luces.

\section{Algunas notas históricas}

Según RÍOS MARTÍNEZ (2015), el origen de los zoológicos se remonta a las civilizaciones antiguas del Viejo Mundo, en donde se puede evidenciar que existían colecciones de animales para ostentación y estudio, como el paradeiso persa, un jardín dentro o cerca del palacio con distintos animales en cautiverio; mientras que en el mundo occidental, en la época medieval existían las llamadas menagerie o "casa de fieras", donde mostrar al pueblo algún oso, lobo o felino en jaulas ambulantes, o para usarlos como modelos por pintores, o como en Roma, para entretener a la nobleza con las luchas entre sus ejemplares. En el siglo xvii, las menageries europeas albergaban animales en edificios de estilos arquitectónicos pertenecientes a los sitios de origen; a finales del siglo XVIII, tras la decadencia de la nobleza, se juntaron varias menageries, que habían sido trasladadas en 1793 al Jardin de Plantes, en Versalles, para estudiar a la naturaleza desde una perspectiva científica; nació así la idea del "jardín zoológico" que en México ya se había desarrollado desde la época prehispánica.

Los ingleses fundaron el jardín zoológico de Londres Regent's Park en 1828, con el propósito de entender la vida silvestre; éste se convertiría en un ejemplo para el desarrollo de futuros zoológicos. En 1890, Carl Hagenbeck, quien era coleccionista de animales salvajes, los exhibió en su propio zoológico, “Tierpark”, en Europa, donde semejaba el hábitat natural de cada ejemplar; para ello empleaba fosos y barreras que se confundían con el mismo hábitat, lo que produjo nuevos estándares de tipo naturalista. Hagenbeck viajó a Estados Unidos con su hermano y participó en el diseño de numerosos zoológicos. En los años 70 se formó en Norteamérica la Asociación de Parques Zoológicos y Acuarios, después llamada $A Z A$, que valora las colecciones de animales para entrenimiento, educación y desarrollo del conocimiento científico, y cuyas estrategias prioritarias, son: avanzar en conservación y cuidado de especies y sus hábitats, educación y vinculación de interna y externa de público y grupos de interés, mejorar los servicios de sus afiliados o miembros, y desarrollar un modelo económico sostenible y robusto (AZA, 2014) (Figura 1).

Modernamente, según lo plantea RÍOS MARTÍNEZ (2015) con acogimiento a lo expresado por GDF (2006), LESLEY et al. (2009) y (MARK y FA, 2009), se puede entender que los zoológicos cumplan la función de museos vivientes, en

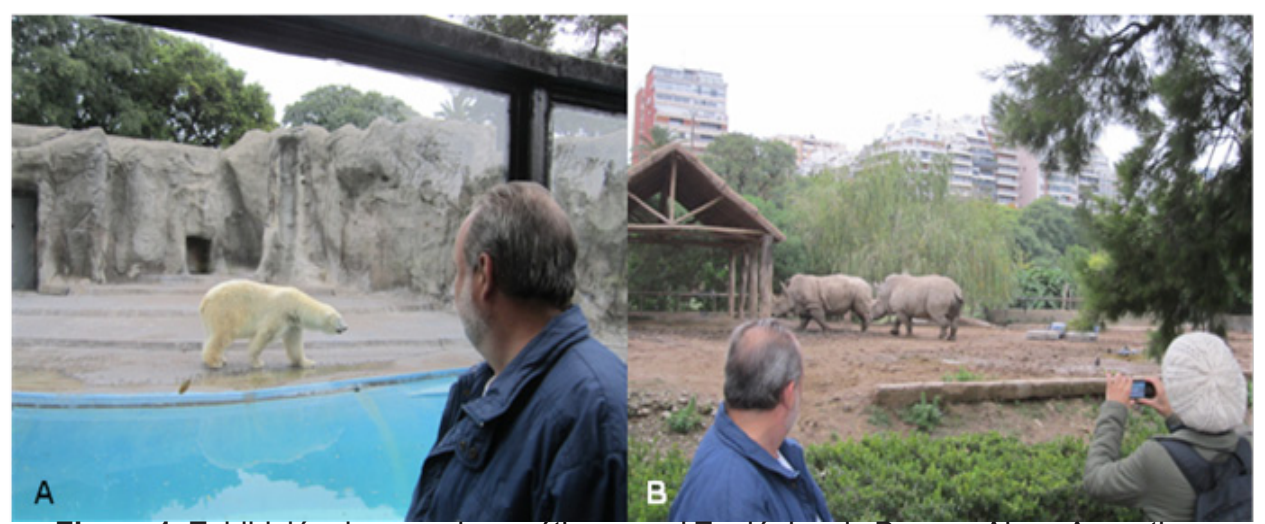

Figura 1. Exhibición de especies exóticas en el Zoológico de Buenos Aires, Argentina. A (Ursus maritimus), B (Diceros bicornis). Fotos: De La Ossa-Lacayo, A. (2013). 
tanto que albergan colecciones de seres vivos, e incluso animales y plantas en peligro de extinción. Asimismo, como todo museo, presentan colecciones bien organizadas que son un valioso aporte a los procesos de conservación global. Muchos zoológicos de Colombia, se encuentran registrados con entidades museales y cuentan con registro SIMCO (OIM, 2013).

\section{Desde el bienestar}

Se puede asumir que el diseño de zoológicos debe atender cuatro enfoques principales: la dirección del zoológico, que exige exhibiciones, investigación, conservación y reproducción de los animales, además de programas educativos, que contribuyan a la eficiencia y viabilidad económica; el visitante, que solicita accesibilidad para observar las colecciones, satisfacción estética y entretenimiento; el cuidador, que busca seguridad física para sí mismo y el animal, además de facilidad para brindar el servicio de aseo y alimentación; y el animal, que demanda refugio, un ambiente natural y poca perturbación (COLLADOS SARIEGO, 1997; RÍOS MARTÍNEZ (2015). (Figura 2).

Pero aspectos variados tienen influencia sobre el bienestar de los animales silvestres bajo cuidados humanos. Por ejemplo, la adaptación, que se define como una capacidad para aceptar las condiciones restrictivas que impone la cautividad y que varían de una especie a otra; algunas especies se reproducen muy bien en cautividad y no muestran habitualmente signos aparentes de falta de bienestar, mientras que otras muy similares viven poco tiempo en cautividad, se reproducen poco o nada, y muestran frecuentemente conductas anormales cuando se mantienen en zoológicos y acuarios (SALAS y MANTECA, 2016).

Al comparar de forma rigurosa la adaptación de varias especies a la cautividad y teniendo en cuenta que existen todavía muchos interrogantes acerca de las diferencias entre especies en cuanto a su capacidad de adaptación al encierro, SALAS y MANTECA (2016) sugieren las siguientes consideraciones:

1. Es frecuente que se preste una especial atención al bienestar de algunas especies que, a su vez, tienen unas capacidades cognitivas especialmente desarrolladas, ya que hay algunas razones para pensar que las especies de mayor desarrollo cognitivo pueden ser especialmente susceptibles a los efectos negativos de la cautividad. Pero es importante recordar que hay otras muchas especies menos 'emblemáticas' que tienen un desarrollo cognitivo similar en algunos aspectos al de las especies consideradas como más 'inteligentes'.

2. Al margen del desarrollo cognitivo, hay otras muchas características que explican las diferencias entre especies en su capacidad de adaptación a la cautividad. Además, es muy posible que estas características varíen en función del grupo taxonómico. Algunos ejemplos de estas características son la distancia media recorrida diariamente en los carnívoros o características de la dieta en los rumiantes.

3. Finalmente, un factor que probablemente resulta clave para explicar parte de las diferencias entre especies es la medida en que se conoce su biología.

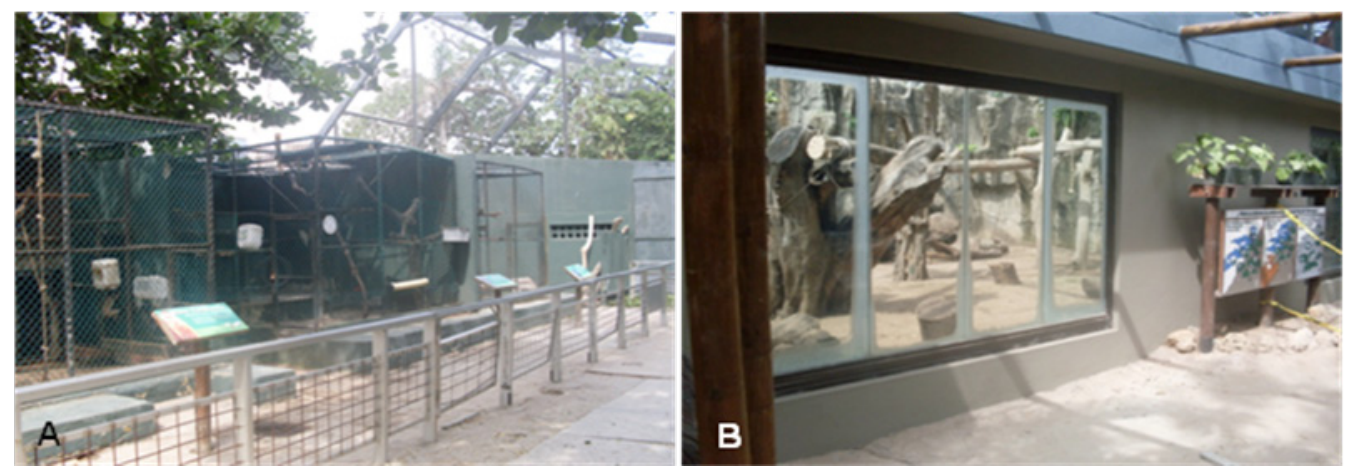

Figura 2. Dos tipos de infraestructura de albergue en el Zoológico de Barranquilla, Colombia (A) Antiguo y (B) Moderno. Fotos: De La Ossa, V.J. (2015). 
En la Tabla 1 se presentan una comparación de las diferencias entre especies de tres grupos taxonómicos respecto de su facilidad de adaptación al cautiverio.

Existe por otro lado el temperamento, definido etológicamente como el nivel de respuesta individual ante estímulos excitatorios, y que puede relacionarse con su bienestar. Las características del temperamento de los animales que se evalúan más frecuentemente son el miedo, la agresividad y la sociabilidad, todas ellas tienen un efecto muy pronunciado sobre su bienestar. La valoración del temperamento de los animales de zoológico tiene numerosas aplicaciones prácticas. Así, se ha demostrado, por ejemplo, en varias especies, que el temperamento de los individuos permite prever su éxito reproductivo, igualmente es valioso cuando se quieren conformar grupos estables de animales (SALAS y MANTECA, 2016). También, está el concepto que indica que los animales poseen personalidad, el cual está ganando cada vez más terreno en la comunidad científica, en especial cuando se investigan sucesos particulares en zoológicos y acuarios, y cuando estos datos etológicos son discutidos con base en la experiencia de quienes hacen el manejo directo de los animales; este rasgo de conducta particular se presenta como un factor importante que puede ser benéfico para los procesos de bienestar que deben ser tenidos en cuenta (WATTERS y POWELL, 2012).

\section{Enriquecimiento.}

De manera ideal se debe tratar, primero de recuperar, ya que en no pocos casos los animales que llegan a los zoológicos, como en sucede en Colombia, en donde la Resolución
2064 de 2010, los señala como entidades en las cuales se pueden disponer los decomisos de fauna silvestre, por lo tanto provienen del tráfico ilegal y su comportamiento se presenta severamente alterado. Segundo, mantener el comportamiento natural de los animales sujetos a cautiverio, que en los zoológicos son parte de los programas de conservación, investigación o educación (WAZA 2005). Es entonces vital que los animales demuestren los comportamientos que generalmente exhiben en su ambiente natural (MASON et al., 2007). No obstante, cuando los animales no cuentan con los estímulos necesarios para regular sus sistemas biológicos, se presentan anormalidades en las respuestas fisiológicas y conductuales, lo que provoca problemas de salud y fallas reproductivas, y denota fallas en el bienestar animal causadas entre otros aspectos, por el ruido, la reducción del espacio o ausencia de elementos paisajísticos complementarios (CASTILLO-GUEVARA et al., 2012).

Como lo señalan (CASTILLO-GUEVARA et al. (2012), las técnicas de enriquecimiento ambiental son usuales en diversos zoológicos, con las cuales se pretende promover el bienestar de los animales cautivos, igualmente se identifican y ofrecen estímulos ambientales para lograr un óptimo desarrollo psicológico y fisiológico (SHEPHERDSON, 1998, MELLEN y MCPHEE, 2001), con lo que se busca reducir o eliminar estereotipias en los especímenes cautivos (Figura $3)$.

Un comportamiento estereotipado o estereotipia, se define, según (MASON, 1991), como cualquier patrón movimiento que es realizado en repetidas ocasiones, relativamente invariable en forma, y sin función u objetivo aparente.

Tabla 1. Comparación de diferencias de adaptación entre especies de tres diferentes grupos taxonómicos de animales silvestres.

\begin{tabular}{|c|c|c|c|}
\hline $\begin{array}{l}\text { Grupo } \\
\text { taxonómico }\end{array}$ & Número de especies & Indicadores de bienestar utilizados & Resultados \\
\hline $\begin{array}{l}\text { Orden } \\
\text { Carnívora }\end{array}$ & 33 & $\begin{array}{l}\text { • Estereotipias. } \\
\text { - Tasa de mortalidad de las crías. }\end{array}$ & $\begin{array}{l}\text { - Las especies que recorren diariamente distancias más grandes se } \\
\text { adaptan peor a la cautividad (CLUBB y MASON, 2007). }\end{array}$ \\
\hline $\begin{array}{l}\text { Suborden } \\
\text { Ruminantia }\end{array}$ & 78 & $\begin{array}{l}\text { - Cociente entre la esperanza de vida } \\
\text { media en cautividad y la longevidad } \\
\text { máxima. }\end{array}$ & $\begin{array}{l}\text { - Las especies ramoneadoras y aquellas para las que no hay guías de } \\
\text { manejo se adaptan peor a la cautividad (MÜLLER et al., 2011). }\end{array}$ \\
\hline $\begin{array}{c}\text { Orden } \\
\text { Psittaciformes }\end{array}$ & 53 & $\begin{array}{l}\text { - Auto-picaje. } \\
\text { - Estereotipias. } \\
\text { - Reproducción. }\end{array}$ & $\begin{array}{c}\text { - Las especies más "inteligentes", las que dedican más tiempo a buscar } \\
\text { el alimento en condiciones naturales, las más amenazadas y las más } \\
\text { especializadas en sus requerimientos ecológicos se adaptan peor a la } \\
\text { cautividad (MCDONALD KINKAID et al., 2014). }\end{array}$ \\
\hline
\end{tabular}




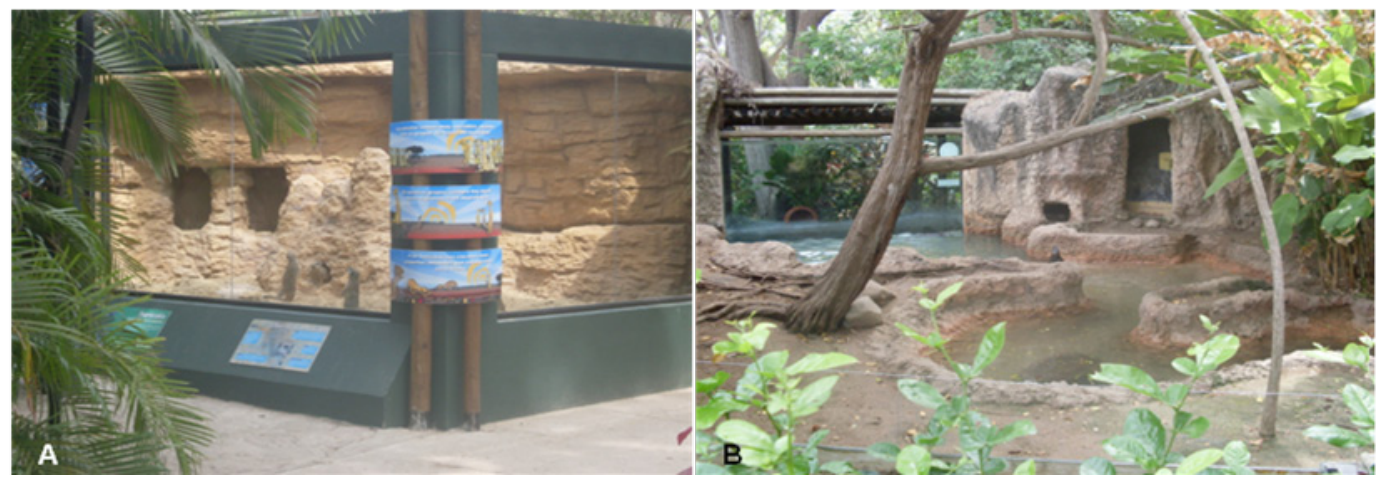

Figura 3. Encierros con elementos de enriquecimiento que ayudan a minimizar conductas estereotipadas en el Zoológico de Barranquilla, Colombia. (A) (Suricata suricatta), (B) (Lontra longicaudis). Fotos: De La Ossa, V.J. (2014).

SHYNE (2006) indica que la frecuencia con que los animales presentan estereotipias es una medida sensible sobre la efectividad de una condición de enriquecimiento. Por ejemplo, factores, tales como el tamaño y complejidad del encierro pueden influenciar o potencializar su presencia (MALLAPUR y CHELLAM, 2002). Por ejemplo, se observan en felinos y otros carnívoros cautivos comportamientos estereotipados, tales como: caminatas repetidas incesantemente, reingestión, regurgitación, coprofagia y aseo excesivo (CLUBB y VICKERY, 2006).

Aun cuando SHYNE (2006) afirma que el enriquecimiento reduce substancialmente los comportamientos estereotipados exhibidos por mamíferos en zoológicos, SALAS y MANTECA (2016) consideran que las respuestas de los animales a los programas de enriquecimiento ambiental dependen de su temperamento: mientras que un estímulo nuevo puede estimular la conducta exploratoria y tener un efecto positivo sobre el bienestar de un individuo poco asustadizo, el mismo estímulo puede tener el efecto contrario o no tener efecto alguno en un individuo muy tímido. Finalmente, hay algunas evidencias que parecen sugerir que el temperamento de los animales condiciona su susceptibilidad a ciertas enfermedades (SALAS y MANTECA, 2016).

No obstante, el enriquecimiento ambiental se utiliza como una buena estrategia para mejorar el bienestar animal que permite reducir conductas estereotipadas, mejorar la actividad e incluso reducir las agresiones en diversas especies de animales en cautiverio (MCPHEE, 2002; BASHAW et al., 2003; SWAISGOOD y SHEPERDSON, 2005; SKIBIEL et al., 2007).

En general y de acuerdo con GARCÍA-PÁRRAGA et al. (2016), es importante tener en cuenta el análisis de las emociones y del comportamiento en cautiverio, ya que al desconocer en detalle cómo interactúan los animales de cada especie en libertad, es complejo interpretar si en cautiverio se están comportando o no de manera natural y, por lo tanto, evaluar su bienestar. Para lo cual se debe orientar el trabajo evaluativo hacia los indicadores directos (observables/medibles), entre los cuales están: los de aspecto o condición corporal, los fisiológicos o los derivados de los registros zoológicos, tales como la incidencia de enfermedad, índices reproductivos o esperanza de vida media, que se convierten en valiosas herramientas a la hora de tomar decisiones de manejo.

\section{Educación ambiental}

Según la SECRETARÍA DEL MEDIO AMBIENTE DEL GOBIERNO DEL DISTRITO FEDERAL DE MÉXICO (2012), la educación ambiental busca incidir en la sensibilidad, los conocimientos y los valores de las personas para promover formas alternativas de relación con el ambiente, de manejo de sus recursos y de servicios ambientales que presta, por medio de la adquisición de conocimientos y habilidades, con la mejora de instrumentos, la difusión de experiencias exitosas de trabajo, contemplando acciones conjuntas con otras instituciones, para crear sinergias y obtener mejores resultados. 
Desde una perspectiva ambientalmente incluyente, la sustentabilidad de un recurso como la fauna silvestre requiere un acercamiento conceptual que vincule diferentes escenarios, entre ellos: la gestión económica, la legislación, las políticas sociales, la ecología y, en especial, la educación ambiental. Ésta última orientada a difundir entre la comunidad el respeto por las diversas formas de vida que coexisten en la naturaleza, según lo señalan MONCADA et al. (2005) citando a FEBRES-CORDERO et al. (1997).

El proceso de educación ambiental debe congregar al mayor número de personas que sea posible, es de factible y de obligatoria realización en especial en las ciudades, como escenario inmediato, ya que posee instalaciones y equipamiento caracterizados por su gran potencial educativo como son los zoológicos, los acuarios, los museos y parques recreacionales (MONCADA et al., 2005). No obstante, para que el proceso sea funcional requiere de una planeación adecuada, debe contar con planes y programas susceptibles de evaluación por objetivos, debe involucrar a los usuarios como parte de la estrategia de construcción y ejecución de las actividades educativas, debe cumplir con la legislación pertinente y los convenios internacionales que hacen parte de las estrategias políticas de conservación de la fauna silvestre, debe buscar apoyo interinstitucional y promover la investigación (SWANAGAN, 2000) (Figura 4).

Ahora, un zoológico no son solo animales cautivos, entre los elementos del establecimiento que brindan importante apoyo a las labores de educación ambiental, según SWANAGAN (2000), están los materiales biológicos conservados: pieles, huesos, plumas, caparazones, por ejemplo. También están los equipos tecnológicos modernos, tales como proyectores y computadores que son de gran importancia (LIANG, 1995; WOODSIDE y KELLY, 1995), además de las labores que en tal sentido ejecutan el personal capacitado que allí labora. Aunque no parezca que exista relación, las labores de educación ambiental tienen estrecha relación con el bienestar animal, un público consciente y bien informado despliega comportamientos notablemente positivos hacia los animales en su visita al zoológico o acuario, y fuera de este sus actitudes frente a la fauna se tornan conservacionistas. En general se puede

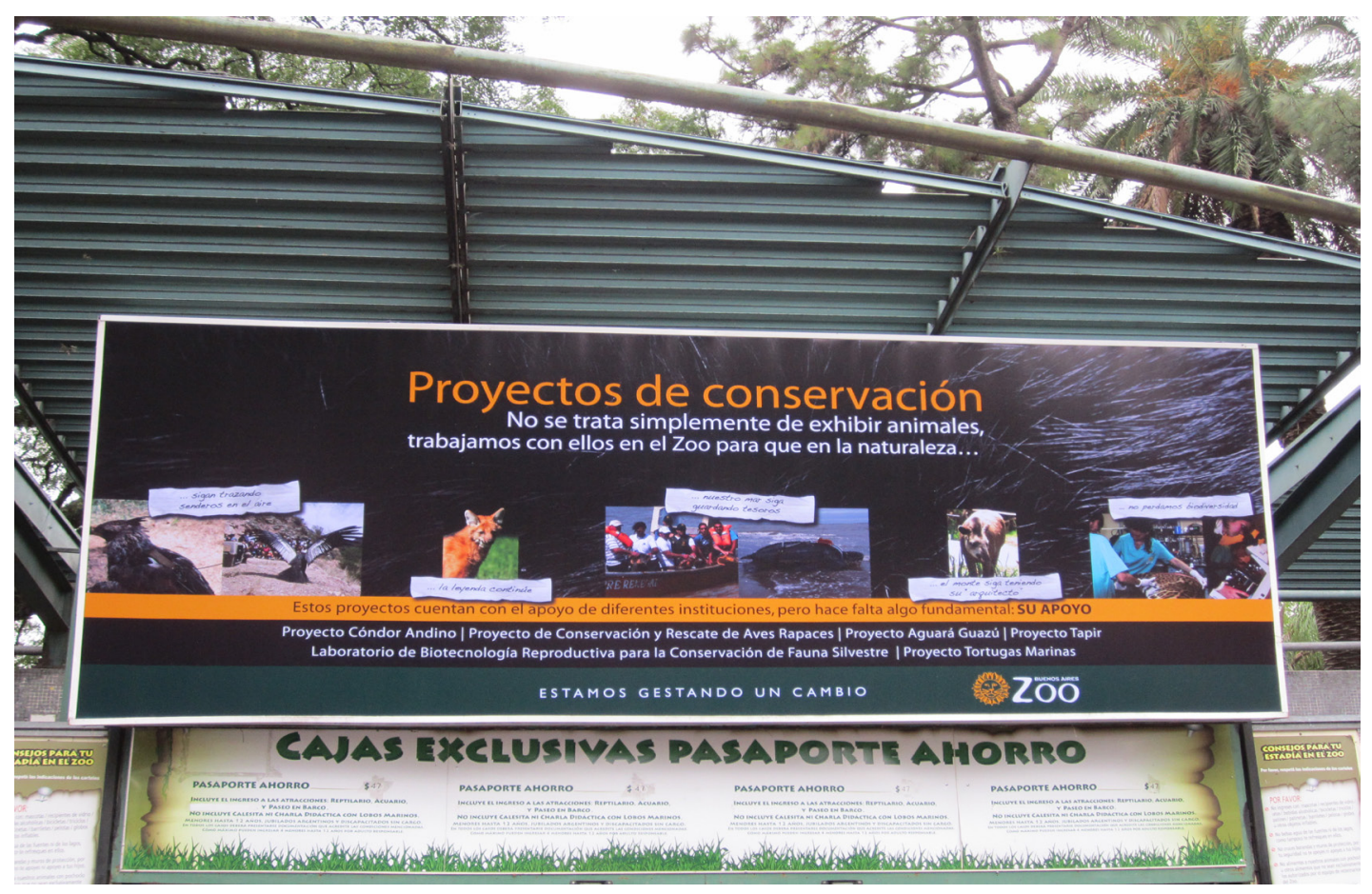

Figura 4. Anuncio de proyectos de conservación, Zoológico de Buenos Aíres, Argentina. Foto: De La Ossa, V.J. (2013). 
afirmar que la apropiación del conocimiento brindado por la educación ambiental en los zoológicos y acuarios, genera mayor valoración por la naturaleza y esto repercute en el bienestar animal de los especímenes en exhibición.

\section{De la señales como medio audiovisual informativo}

VERDI (2014) conceptúa que debido a que normalmente se piensa que los visitantes pueden tener un efecto negativo en el comportamiento de los animales, algunos zoológicos han diseñado señalización creativa para abordar este problema (Figura 5), lo que llevó a los visitantes a ser más tranquilos y más respetuosos (MAPLE y PERDUE, 2013); por lo que se sugiere, por ejemplo, que los felinos y los primates tengan actividades de enriquecimiento ambiental como el entrenamiento para disminuir los periodos de inactividad en presencia de los visitantes y así crear una relación positiva con los mismos (MILLER, 2012).

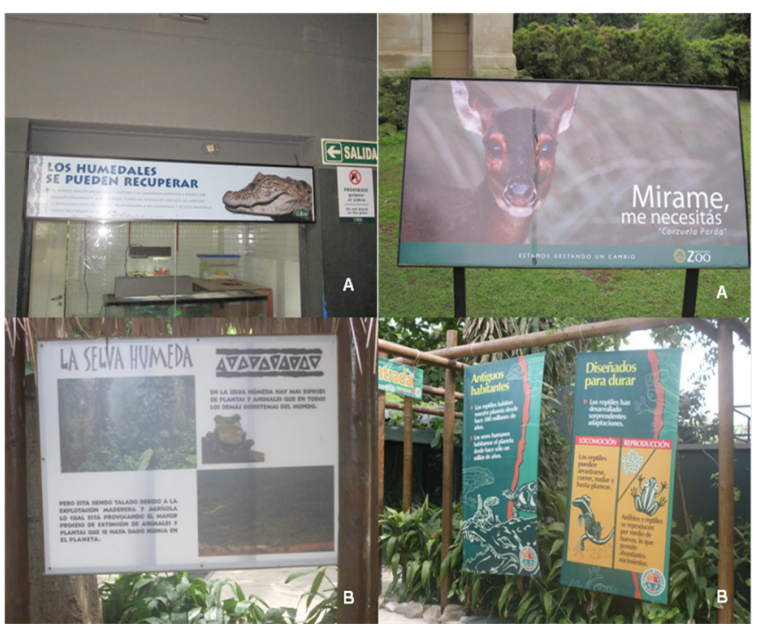

Figura 5. Señales creativas. (A) Zoológico de Buenos Aíres, Argentina. (B) Zoológico de Barranquilla, Colombia. Fotos: De La Ossa, V.J. (2013).
Entre los elementos que hay que considerar para la señalización al interior de un zoológico, ya que se puede inferir que tiene relación con el bienestar animal, puesto que de la información ofrecida se esperan conductas apropiadas de los visitantes que repercuten en el confort de los animales bajo cuidado humano, se tienen, según REIDL MARTÍNEZ et al. (1999): 1. Direccionales o de ubicación; 1.2. Identificativas de lugares; 1.3. Informativas; y 1.4. Restrictivas o prohibitivas (Figura 6).

$<<E l$ bosque está en llamas y mientras todos los animales huyen despavoridos para salvar su vida, un pequeño colibrí vuela en dirección contraria, ha ido al río para recoger agua para verterla sobre el fuego. "Estás loco, ¿acaso crees que con ese pequeño pico vas a apagar el incendio?”, le pregunta el león. Responde el pajarito, "Yo sólo sé que estoy haciendo mi parte >> (BETINHO Citado por IVANOVIC BARBEITO, 2011).

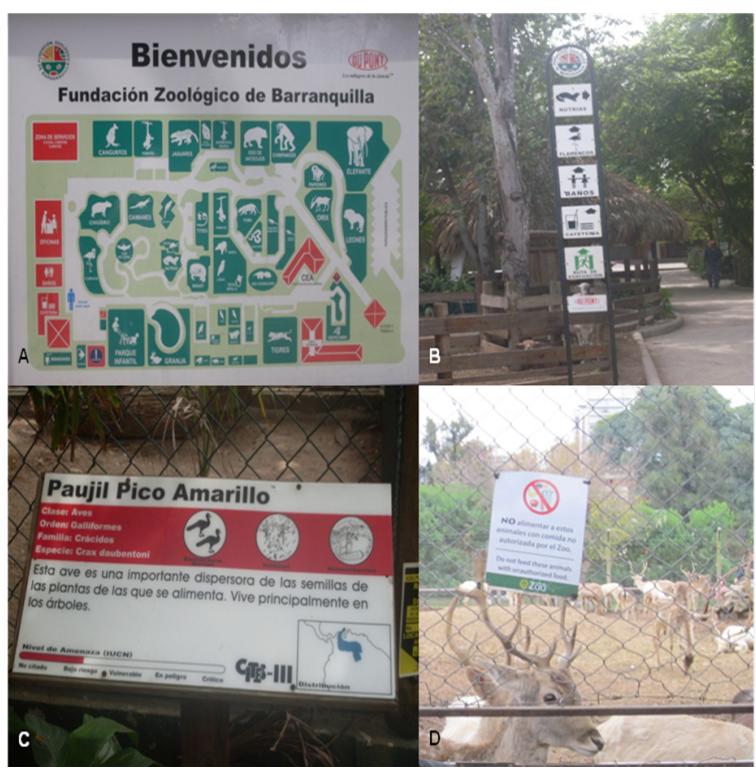

Figura 6. Tipos de señales internas del zoológico de Barraquilla, Colombia: (A) Direccionales, (B) Identificativas y (C) Informativas; Zoológico de Buenos Aíres, Argentina: (D) Restrictivas o prohibitivas. Fotos: De La Ossa, V.J. (2013). 


\section{Referencias}

AZA - ASSOCIATION OF ZOOS AND AQUARIUMS. 2014. Strategy Plan 2015-2017. Disponible en: https://www.aza.org/strategic-plan.Consultado: 09-11-2016.

BASHAW, M.; BLOOMSMITH, M.; MAPLE, T. 2003. To hunt or not to hunt? A feeding enrichment experiment with captive large felids. Zoo Biology 22:189-198.

BECERRA, M.T. 2003. Lineamientos para el manejo sostenible de sistemas de aprovechamiento de recursos naturales in situ. Instituto de Investigación de Recursos Biológicos Alexander von Humboldt. Bogotá, Colombia.

CASTILLO-GUEVARA, C.; UNDA-HARP, K.; LARA, C.; SERIO-SILVA, J.C. 2012. Enriquecimiento ambiental y su efecto en la exhibición de comportamientos estereotipados en jaguares (Panthera onca) del parque zoológico "Yaguar Xoo", Oaxaca. Acta Zoológica Mexicana 28 (2):365-377.

CLUBB, R.; MASON, G. 2007. Natural behavioural biology as a risk factor in carnivore welfare: how understanding species differences could help zoos readesign enclosures. Applied Animal Behaviour Science 102:303-328.

CLUBB, R.; VICKERY, S. 2006. Locomotory Stereotypies in Carnivores: Does Pacing Stem from Hunting, Ranging or Frustrated Escape?. Págs. 58-85. En: Mason, G.; Rushen, J. (Eds.). Stereotypic animal behaviour. Fundamentals and Applications to Welfare. Second ed. CABI international. Londres.

COLLADOS SARIEGO, G. 1997. EI Rol de los Zoológicos Contemporáneos. Disponible en: http://www. zoolex.org/publication/collados/collados.pdf. Consultado: 10-07-2015.

CORPONARIÑO - CORPORACIÓN AUTÓNOMA REGIONAL DE NARIÑO. 2016. Glosario de términos. Disponible en: http://corponarino.gov.co/modules/wordbook/entry.php?entrylD=197 . Consultada: 0511-2016.

FEBRES-CORDERO, M. E.; LUQUE, L.; ARANGUREN, J.; VELASCO, F.J. 1997. La Educación Ambiental. Paradigma del tercer milenio. D.G.S.E.A.P.C. Caracas, Venezuela.

GARCÍA-PÁRRAGA, D.; LÓPEZ DE LA OSA-ESCRIBANO, P.; FERNÁNDEZ-LÁZARO, G.; ALONSOGARCÍA, E. 2016. Educación sobre bienestar de animales silvestres en aquariums y zoos (ex situ). Revista de Docencia Veterinaria 1:124-125.

GDF - Dirección General de Zoológicos de la Ciudad de México, 2006. Centros de Conservación del Siglo xxi. Memorias 2001-2006. Gobierno del Distrito Federal, Secretaria del Medio Ambiente. México.

IVANOVIC BARBEITO, M. 2011. Bioética animal. Un decálogo animalista. Revista de Bioética y Derecho 22: $56-66$.

IVANOVIC BARBEITO, M. 2013. Derechos animales versus holocausto animal. Viento sur 126:6-79.

LESLEY A.D.; BONNER, J.P.; WEST, C. 2009. In situ and ex situ conservation: blurring the boundaries between zoos and the wild. Págs. 221. En: Zimmermann, A.; Hatchwell, M.; Dickie, L.A.; West, C. (Eds.). Zoos in the 21st Century. Cambridge: Cambridge University Press.

LIANG, M.H. 1995. The magic of touch!. Journal of the International Association of zoo educators 32:19-24. 
MALLAPUR, A. 1999. Environmental influences on space utilization and the activity budget of captive leopards (Panthera pardus fusca) in five zoos in Southern India. Tesis de Maestría. Saurashtra University. India.

MALLAPUR, A.; CHELLAM, R. 2002. Environmental influences on stereotypy and the activity budget of Indian leopards (Panthera pardus) in four zoos in Southern India. Zoo Biology 21: 585-595.

MAPLE, T.; PERDUE, B. 2013. Zoo animal welfare. Springer. London.

MARK, R.S.P.; FA, J.E. 2009. Reintroductions from zoos: a conservation guiding light or a shooting star?" Págs. 156. En: Zimmermann, A.; Hatchwell, M.; Dickie, L.A.; West, C. (Eds.). Zoos in the 21st Century.. Cambridge: Cambridge University Press.

MASON, G.J. 1991. Stereotypies: a critical review. Animal Behaviour 41:1015-1037.

MASON, G.; CLUBB, R.; LATHAM, N.; VICKERY, S. 2007. Why and how should we use environmental enrichment to tackle stereotypic behaviour? Applied Animal Behaviour Science 1002:163-188.

MCDONALD KINKAID, H.; VAN ZEELAND, Y.; SCHOEMAKER, N.; KINKAID, M.; MASON, G. 2014. Risk factors for poor welfare in Psittaciformes: do intelligence and foraging behaviour predict vulnerable parrot species? Proceedings of the 48th Congress of the International Society for Applied Ethology. Vitoria-Gasteiz.

MCPHEE, E. 2002. Intact carcasses as enrichment for large felids: Effects on on- and off-exhibit behaviors. Zoo Biology 21:37-47.

MELLEN, J.; MCPHEE, M.S. 2001. Philosophy of environmental enrichment: past, present, and future. Zoo Biology 20:211-226.

MILLER, L. 2012. Visitor Reaction to Pacing Behavior. Zoo Biology 31:242-248.

MONCADA, J.A.; DÍAZ, E.; ARANGUREN, J.; PELLEGRINI, N. 2005. Características de los departamentos de educación en las instalaciones zoológicas venezolanas y propuestas para su gestión. Investigación y Postgrado 20 (1):175-205.

MÜLLER, D.W.H.: LACKEY, L.B.; STREICH, W.J.; FICKEL, J.; HATT, J.M.; CLAUSS, M. 2011. Mating System, feeding type and ex situ conservation effort determine life expectancy in captive ruminants. Proceedings of the Royal Society B 278:2076-2080.

OIM - OBSERVATORIO IBEROAMERICANO DE MUSEOS. 2013. Marco conceptual común para la elaboración del registro de museos Iberoamericanos. Informe con la recopilación de datos, registros y censos de museos lberoamericanos y primera propuesta teórica para el futuro registro de museos. Disponible en: http://www.ibermuseus.org/wp-content/uploads/2014/09/OIM_RMI_INFORME_PREVIO MARCO CONCEPTUAL-ALTA.pdf. Consultado: 09-11-2016.

REIDL MARTÍNEZ, L.; SIERRA OTERO, G.; MENDIETA AZNAR, R. 1999. El zoológico de San Juan de Aragón desde el punto de vista psicosocial. Universidad Nacional Autónoma de México, Facultad de Psicología. Gobierno del Distrito Federal. Dirección General de Zoológicos de la ciudad de México. México.

RÍOS MARTÍNEZ, A. 2015. Los zoológicos en la arquitectura de paisaje. bitácora arquitectura 31:14-21. 
SALAS, M.; MANTECA, X. 2016. ¿Hay animales que se adaptan mejor que otros a la cautividad?. Ficha técnica sobre bienestar en animales de zoológico. Disponible en: www.zawec.org.

SÁNCHEZ, S.; LOZADA, N.; TORRES. A. 1997. Los zoológicos en la Educación Ambiental. Instituto de Historia Natural. Chiapas, México.

SECRETARÍA DEL MEDIO AMBIENTE DEL GOBIERNO DEL DISTRITO FEDERAL. 2012. La educación ambiental como eje transversal para una nueva cultura ambiental en la ciudad de México. Gobierno del distrito federal. Libros blancos: 2016-2012. México.

SHEPHERDSON, D. 1998. Tracing the path of environmental enrichment in zoos, Págs. 1-12. En: Shepherdson, D.; Mellen, J.; Hutchins, M. (Eds.). Second nature: environmental enrichment for captive animals. Washington: Smithsonian Institution Press.

SHYNE, A. 2006. Meta-analytic review of the effects of enrichment on stereotypic behavior in zoo mammals. Zoo Biology 25:317-337.

SKIBIEL, A.; TREVINO, H.; NAUGHERZ, K. 2007. Comparison of several types of enrichment for captive felids. Zoo Biology 26:371-381.

SWAISGOOD, R.R.; SHEPHERDSON, D.J. 2005. Scientific approaches to enrichment and stereotypies in zoo animals: what's been done and where should we go next? Zoo Biology 24:499-518.

SWANAGAN, J. 2000. Factors influencing Zoo visitors, conservation attitudes and behavior. The Journal of Environmental Education 31 (4):26-31

TUBIELLO , F.N.; SALVATORE, M.; ROSSI, S.; FERRARA, A.; FITTON, N.; SMITH, P. 2013. The FAOSTAT database of greenhouse gas emissions from agriculture. Environ. Res. Lett. 8 doi:10.1088/174 8-9326/8/1/0150 09.

TUBIELLO, F.N.; SALVATORE, M.; CÓNDOR GOLEC, R.D.; FERRARA, A.; ROSSI, S.; BIANCALANI, R.; FEDERICI, S.; JACOBS, H.; FLAMMINI, A. 2014. Agricultura, silvicultura y otros usos de la tierra emisiones por fuentes y absorciones por sumideros. Análisis 1990-2011. FAO - Organización de las Naciones Unidas para la Agricultura y la Alimentación. Roma.

UNESCO. 1977. DECLARACIÓN universal de los derechos de los animales. Disponible en: http:// academic.uprm.edu/rodriguezh/HTMLobj-139/DERECHOS_ANIMAL_UNESCO.pdf. Consultada: 3009-2016.

VÁLDES, V.V. 2008. Prácticas de manejo en la conservación Ex Situ y su relación con la sostenibilidad ambiental. Tecnología en Marcha 21 (1):152-160.

VASTA, M.C. 2013. Modos de ver. Abordajes epistemológicos para el estudio del Jardín Zoológico de Buenos Aires. Págs. 514-522. En: $1^{a}$ Jornada de Investigadores en Formación. JIF-FADU. Argentina.

VERDI, O.S. 2014. El uso del entrenamiento como parte de un programa de bienestar para Pumas (Puma concolor) en cautiverio. Disponible en: http://congreso.fmvz.unam.mx/pdf/Memorias\%20de\%20 carteles/EL\%20USO \%20DEL\%20ENTRENAMIENTO $\% 20$ COMO $\% 20$ PARTE\%20DE\%20UN\%20 PROGRAMA\%20DE\%20\%20BIENESTAR\%20\%20PARA\%20PUMAS\%20(Puma\%20concolor)\%20 EN\%20CAUTIVERIO.pdf. Consultado: 21-08-2016.

WATTERS, J.V.; POWELL, D.M. 2012. Measuring Animal Personality for Use in Population Management in Zoos: Suggested Methods and Rationale. Zoo Biology 31 (1):1-12. 
WAZA - World Association of Zoos and Aquariums. 2005. The World Zoo Conservation Strategy: Building a Future for Wildlife. World Aquaria and Zoos Association Executive Office, Bern.

WINT, W.; ROBINSON, T. 2007. Gridded livestock of the world. FAO - Organización de las Naciones Unidas para la Agricultura y la Alimentación. Roma.

WOODSIDE, D. P.; KELLY, J.D. 1995. The development of local, national and international zoo-based education programmes. International Zoo Year Book 34:231-246. 